\title{
Nuevas perspectivas de la investigación dirigida en la Escuela de Ciencias de la Administración
}

\author{
Mag. Ileana Ulate Soto* \\ Mag. Elizarda Vargas Morúa**
}

Recibido: Marzo 2012 • Aceptado: Mayo 2012

\begin{abstract}
RESUMEN
Mediante este trabajo, se exponen las principales características de la asignatura en línea denominada Investigación Dirigida, código 4010, de la Escuela de Ciencias de la Administración de la Universidad Estatal a Distancia. Este curso se encuentra en el bloque final de asignaturas y fue diseñado en el año 2009; entre los cambios realizados se pueden señalar: la oferta en línea a través de la plataforma virtual Moodle, la participación de un tutor coordinador y de un tutor especialista responsable de velar por la correcta elaboración del proyecto de investigación, así como de la valoración y pertinencia del énfasis elegido. Los cambios surgieron por la necesidad de reducir las dificultades que manifestaban los estudiantes en la elaboración de su tesis de grado y, de esta forma, potenciar los diferentes recursos de mediación empleados. Se presenta un análisis de indicadores donde se valora la opinión de los estudiantes con respecto al impacto que han tenido los diferentes recursos de mediación empleados en el desarrollo de su proyecto de investigación.
\end{abstract}

Palabras clave: investigación dirigida, diseño de curso, plataforma, tutor especialista, coordinador de curso, recursos didácticos.

\section{ABSTRACT}

Through this paper we describe the main features of the online course "Directed Research", Code 4010 of the School of Management Sciences. This course is in the final block of subjects and was redesigned in 2009. Changes include online access through the Moodle virtual platform, the participation of a tutor coordinator and a specialist, who are responsible for ensuring the proper preparation of student research projects, their evaluation, and the relevance of

* Licenciada en Docencia. Máster en Administración de Empresas de la Universidad Latinoamericana de Ciencia y Tecnología. Egresada del Programa de Doctorado en Administración de la Universidad Estatal a Distancia (UNED). Encargada de la Cátedra de Investigación de la Escuela de Ciencias de la Administración de la UNED. E-mail: iulate@uned.a.c.cr

** Licenciada de la Facultad de Derecho de la Universidad de Costa Rica. Máster en Administración de Empresas de la Universidad Latinoamericana de Ciencia y Tecnología. Tutora en la Cátedra de Investigación de la Escuela de Ciencias de la Administración de la Universidad Estatal a Distancia (UNED). 
the emphasis chosen. The changes arose from the need to minimize student difficulties with developing their thesis, and thus utilize all mediation resources available. Below is an analysis of indicators that evaluates the views of students about of the different mediation resources used in the development of their research project.

Key words: targeted research, course design, specialist tutor, course coordinator, teaching resources.

\section{Antecedentes}

En la Escuela de Ciencias de la Administración, antes del año 2009, si un estudiante optaba por la modalidad de tesis para obtener el grado de licenciado en Administración, matriculaba la asignatura de código 4010, denominada Investigación Dirigida; en esta se le ofrecía, en un semestre, elaborar los tres primeros capítulos de la tesis y en una segunda parte, mediante el curso 460, realizar los últimos tres capítulos.

En el curso 4010, se le brindaban cuatro clases presenciales, en las cuales se le informaba y capacitaba en los aspectos metodológicos considerados esenciales para el buen desarrollo de la tesis. Asimismo, a los estudiantes se les solicitaba avances de los tres primeros capítulos del proyecto, previamente aprobado por el encargado de programa respectivo. En este momento, la realimentación ofrecida era más de corte metodológico, pues el mismo encargado de cátedra era el responsable del curso; además, se aducía que, en el curso siguiente, el especialista ofrecería la dirección necesaria en el contenido especializado.

La práctica antes descrita generaba grandes atrasos en los estudiantes, dado que cuando al especialista le correspondía iniciar la dirección de los trabajos, usualmente se requería hacer grandes ajustes en el trabajo realizado hasta el momento. Por tal razón, en el Consejo de Escuela del 26 de agosto del 2009, se propusieron los cambios y el rediseño de la modalidad impartida hasta ese momento.

En ese año 2009, siendo conscientes de la necesidad de modernizar y realizar cambios en beneficio de una mejor orientación y dirección a los estudiantes, se diseñó el curso del código 4010, siempre bajo el mismo nombre, es decir, Investigación Dirigida.

Esta asignatura, al igual que su predecesora, se ubica al final del bloque de nivel de licenciatura y tiene como requisito haber aprobado el plan de estudios correspondiente a dicho nivel o matricular simultáneamente las dos últimas materias que el educando tenga pendientes de aprobación. 
El propósito de esta asignatura se podría resumir en la realización de un trabajo mediante el cual el estudiante aplique los conocimientos teóricos y prácticos adquiridos durante el estudio en cualquiera de los énfasis de la carrera de Administración de Empresas. Para ello, el educando debe proponer su anteproyecto ante la Comisión de Trabajos Finales de Graduación, para que esta, de conformidad con lo establecido en el Reglamento de Trabajos Finales de Graduación, analice y avale la solicitud y el anteproyecto.

Durante esta asignatura, el estudiante desarrollará al lado de un tutor los tres primeros capítulos de la investigación. Este proceso concluye cuando, el semestre siguiente, el alumno matricula el curso con el código 460 y culmina, así, el proceso realizando los últimos tres capítulos y la defensa final de la tesis ante un tribunal examinador.

\section{Referencia general del modelo}

Como se ha descrito, este no es un curso típico; cada alumno matricula y desarrolla, de conformidad con su énfasis, su propio proyecto de investigación con ayuda de un tutor asignado según el énfasis. La parte común a todos los estudiantes, como son las temáticas referidas al tipo y enfoque de investigación, pasos para elaborar el marco teórico y la metodología, se desarrollan a través de la plataforma, la cual sirve de apoyo.

El montaje en la plataforma permite aprovechar las ventajas que ofrece la educación en línea, entre ellas: la posibilidad de poner a disposición de los alumnos un amplio volumen de información, facilitar la actualización de la información y de los contenidos, flexibilizar la información (independientemente del espacio y el tiempo en el cual se encuentran el profesor y el estudiante), facilitar la autonomía del alumno, ofrecer diferentes herramientas de comunicación sincrónica y asincrónica para estudiantes y docentes, permitir que en los servidores pueda quedar registrada la actividad realizada por los estudiantes, entre otros (Cabero, 2006).

También, se rescata la presencialidad, dado que tal y como lo indican los autores Bañuelos y Barrón $(2005$, p. 53) refiriéndose a la educación en línea, "la educación debe ser abarcativa e incluso considerar que no debe descartarse la presencialidad, sino todo lo contrario". Al respecto se ha considerado que los estudiantes a distancia carecen de experticia en la presentación de trabajos orales, y siendo que la defensa de su tesis debe realizarse ante un jurado calificador, se incorpora la presentación oral del avance realizado en la tesis, respecto a 
los tres primeros capítulos, como trabajo final para concluir el curso.

En esta reunión presencial y final, se busca que el tutor culmine con el proceso de orientación, ayuda o consejo al alumno con el fin de que este alcance sus objetivos, resuelva dudas y, en definitiva, logre "superar el aislamiento que estos entornos producen en el individuo" (Llorente, 2006, p. 7).

Otro aspecto en el que se ha puesto especial importancia es en los contenidos que se brindan a los estudiantes. Además del libro base, se dispone de materiales complementarios, los cuales buscan cumplir con una de las variables llamadas críticas en la educación a distancia, es decir, los contenidos. Estos deben cumplir con un nivel de calidad (en el sentido de pertinentes y relevantes), cantidad (para que el volumen sea adecuado a las características del grupo y los objetivos que se persiguen) y estructuración adecuada que permita una buena presentación del mensaje (Cabero, 2006).

En fin, se espera que, como lo señalan ciertos autores de educación en línea, esta asignatura incluya interacciones y no esté constituida por la mera distribución de materiales, pero sí permita aprovechar las facilidades y el enriquecimiento que ofrece este medio (González-Videgaray, 2007).
En cuanto al coordinador, su participación cumple en cierta medida con las actividades del llamado "tutor virtual" (Llorente, 2006), por ejemplo: en la realización de actividades formativas específicas, al incorporar y modificar nuevos materiales en el entorno formativo, mantenerse en contacto con el administrador del sistema, y otras como la coordinación entre tutores y alumnos, los cronogramas, las dudas en cuanto a formalidades del trabajo de investigación. Sin embargo, actividades como la capacitación de los alumnos en el funcionamiento técnico del entorno telemático de formación o bien dar consejos y apoyos técnicos, se realizan con el acompañamiento de los funcionarios del Programa de Aprendizaje en Línea (PAL), quienes realizan algunas de estas funciones a solicitud del coordinador.

Con el diseño e implementación de esta asignatura se ha pretendido, como lo señala Díaz (2011), buscar un modelo pedagógico que permita tanto aprovechar todas las posibilidades existentes como que los cambios nos posibiliten dar respuesta a los requerimientos educativos que van surgiendo.

A continuación, se describen con mayor detalle las modificaciones realizadas con la nueva propuesta. 


\section{Cambios realizados en el nuevo diseño}

En el actual diseño, se incorporaron cambios con el fin de favorecer el aprendizaje significativo de los alumnos; dentro de estos, se pueden mencionar los que aparecen en los siguientes apartados.

\section{La asignatura se ofrece en plataforma virtual}

En este caso particular, se utiliza la plataforma Moodle. Esta es un software libre, sin costo para la Universidad y de muy fácil uso, según lo manifestaron los propios estudiantes (ver al respecto el resultado del cuestionario aplicado). Además, la plataforma, de conformidad con las productoras académicas, permite incluir grupos estudiantiles grandes.

Para el desarrollo de tal herramienta, se organizaron cinco módulos y se utilizó como libro base el de Metodología de la investigación, de los autores Hernández, Fernández y Baptista (2010). En la plataforma, los estudiantes deben efectuar prácticas virtuales para corroborar la realización de las lecturas. También, participan en foros y blogs con temáticas como el tipo y el enfoque investigativos que realizarán, los alcances de esta y la estructura inicial de la investigación. En esta primera parte, se busca que el educando conozca los lineamientos teórico-metodológicos y partir así de una base común de información entre los estudiantes. Finalmente, en los módulos tres, cuatro y cinco se brindan las bases para la elaboración de los tres primeros capítulos de su tesis: la introducción, el marco teórico y el marco metodológico, respectivamente.

Al ser un curso teórico-práctico, se desarrollarán actividades que permitan la comprobación de la teoría estudiada; por tanto, se propone el uso de la plataforma, la cual permite una continua interactividad profesor-alumno. Esta servirá de apoyo didáctico, facilitando obtener los conceptos y procedimientos de una manera fácil. También, con este tipo de estrategias se pretende facilitar los procesos de socialización y realimentación por parte de los alumnos, con el fin de enriquecer el quehacer de la investigación y el desarrollo profesional.

El número de tutores dependerá de la cantidad de estudiantes matriculados, siendo que cada tutor contratado atiende un máximo de cinco estudiantes por semestre.

\section{El coordinador}

La plataforma virtual está a cargo de un tutor coordinador de la Cátedra de Investigación, quien tendrá a cargo la orientación de los temas. Le corresponderá la 
realimentación, calificación de algunas de las tareas como, por ejemplo, el mapa conceptual del marco teórico y el cuadro de variables correspondiente al capítulo del marco metodológico. Además, comprobará la realización de las lecturas obligatorias, coordinará el nombramiento del tutor según el énfasis del alumno, evacuará dudas relativas a la metodología y el formato de presentación de la tesis y, en general, velará por que el proceso se desarrolle sin contratiempos para los estudiantes. También, será la persona responsable de entregar las notas a la encargada de cátedra para el respectivo trámite en Registro.

\section{Tutores por énfasis}

Otro cambio importante en este diseño es el nombramiento de tutores por cada énfasis, llámese por ejemplo: Contabilidad, Mercadeo, Finanzas, Recursos Humanos, Producción. Estos tutores son contratados por servicios profesionales para revisar los tres primeros capítulos de la tesis. El tutor deberá leer y calificar los capítulos 1,2 y 3 , con base en unas rúbricas creadas para tal efecto. Este tutor es nombrado por la Cátedra de Investigación, de acuerdo con el énfasis del estudiante y será el responsable de guiarlo y asesorarlo en la elaboración del trabajo de investigación, según el área escogida; para ello, estimulará el intercambio de ideas por medio de la plataforma virtual, revisará y evaluará los avances de la tesis correspondientes a los tres primeros capítulos -que se presentan a lo largo del semestre- así como la exposición del informe final de tales apartados.

\section{Recursos de apoyo}

Se incorporaron diversos recursos de apoyo para el estudiante. En tanto ser autónomo y autorregulado, cada educando deberá realizar las lecturas asignadas y propias para su investigación, para luego relacionarlas con su propio proyecto investigativo, el cual se desarrollará en forma individual.

Dentro de los recursos de apoyo se ofrecen:

Libro base de metodología. Corresponde a los autores Hernández, Fernández y Baptista (2010) y se titula Metodología de la investigación. Este desarrolla una descripción detallada de los enfoques cuantitativo y cualitativo de la investigación. Abarca temas relacionados con el surgimiento de un proyecto investigativo, el planteamiento del problema de investigación, la elaboración del marco teórico, la selección de la muestra y el reporte de los resultados, entre otros. 
Normas APA. Dentro de los recursos de los módulos, se incorporan las normas APA, sexta edición, y se solicita a los estudiantes ajustar su trabajo a estas.

Plagio y derechos de autor. Se incorporan lecturas respecto a plagio y derechos de autor, así como algunas noticias de ejemplos de plagios publicitados en los medios de comunicación. Se insta a los estudiantes a ser rigurosos con las citas bibliográficas y a concientizarse de las consecuencias de no respetar la normativa universitaria en ese sentido.

Biblioteca. Se motiva a los educandos a recurrir a la biblioteca, a hacer uso de la biblioteca virtual y de préstamos interbibliotecarios. Se les ofrecen los manuales de Ebsco y Proquest, a fin de proveerles nuevos medios para la obtención de material científico.

Rúbricas. El estudiante tiene acceso a las rúbricas de calificación, en las cuales se contempla el buen uso del lenguaje, puntualidad en la entrega pero, ante todo, en el cumplimiento con los objetivos de cada capítulo, por ejemplo: para el capítulo primero, se califica el respeto por el formato establecido, el apego a las normas APA, si el título refleja el problema por investigar, la formulación del problema, la redacción de los objetivos de manera coherente y con una estructura adecuada, la importancia y justificación del trabajo, la indicación de las limitaciones del problema, entre otros.

Agenda por módulos. Se elaboró un cronograma por módulo para ayudar al alumno en la organización y desarrollo de cada actividad así como en la utilización de los recursos, de tal manera que conozca con claridad las lecturas y actividades de cada semana. Con ayuda de la agenda, se facilita la coordinación entre el coordinador del curso y los tutores, así como hacia los estudiantes.

Otras lecturas. Entre las lecturas complementarias, se pueden citar las orientaciones, instrucciones para participar en foros, formato de la tesis, guía para elaborar los objetivos y el Reglamento de Trabajos Finales de Graduación de la Universidad.

Safe Assign. Cuando se tienen dudas acerca de si el trabajo es auténtico e inédito, se utiliza la herramienta Safe Assign con el fin de corroborar su legitimidad. 


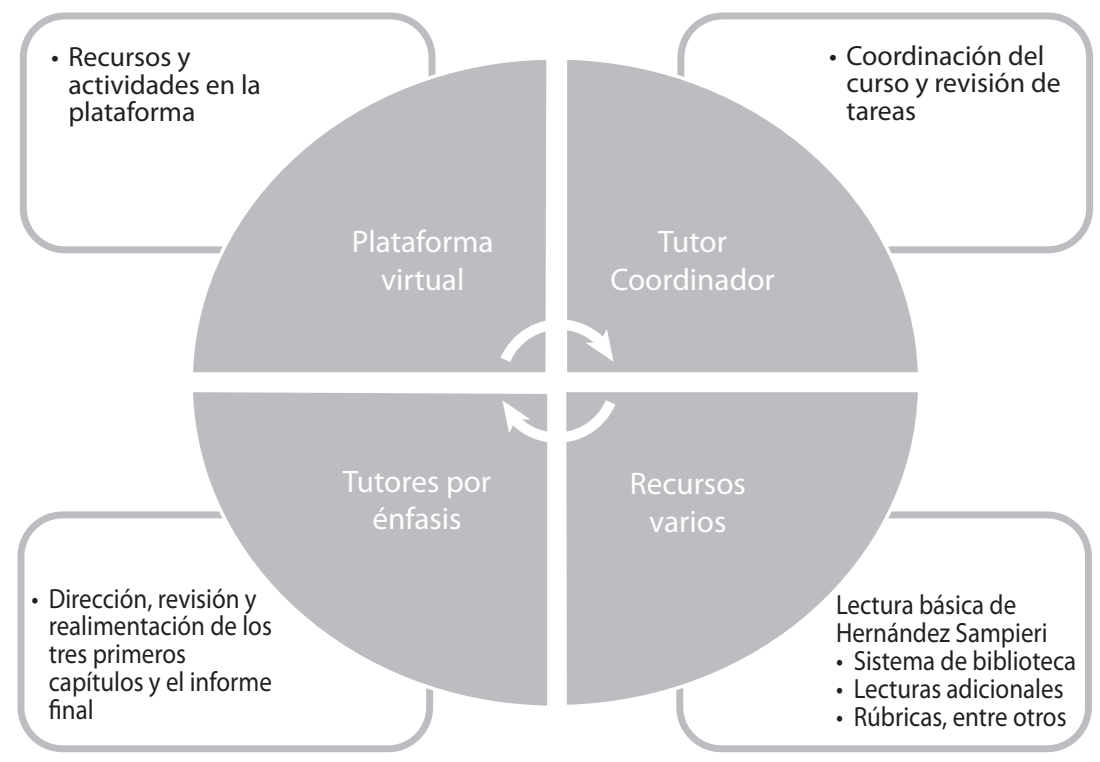

Figura 1. Cambios estructurales del curso de investigación dirigida. Fuente: Elaboración propia, Ulate y Vargas (2011).

\section{Actividades realizadas}

Se incorporaron actividades previas al curso, que se desglosan a continuación.

\section{Capacitación}

En este momento, se está en un proceso de capacitación a los profesores en la plataforma Moodle (un $50 \%$ ya fue capacitado) y se los autorizó para que directamente accedieran a la plataforma y realizaran la calificación respectiva. La tutora coordinadora, previo a ello, recibió capacitación del CECED con respecto a mapas conceptuales, plagio, WebCT, Blackboard y Moodle.

\section{Preselección de temas}

Se realiza una preselección de los temas de investigación. Los alumnos deben entregar un anteproyecto y la Comisión de Trabajos de Investigación, cumpliendo con el nuevo Reglamento de Trabajos Finales de Graduación, evaluará su pertinencia o no. El anteproyecto debe entregarse un mes antes de la matrícula; este puede ser aprobado 
o reprobado, o bien se lo aprueba pero sujeto a modificaciones. Finalmente, el encargado de programa correspondiente le autorizará la matrícula al estudiante.

También, se incorporaron actividades para realizar durante el desarrollo del programa. Entre ellas, las siguientes:

\section{Actividades a través de la plataforma}

Se participa en un foro de discusión de algunos de los temas del curso; en blogs sobre la construcción de conceptos básicos, en los cuales deben identificar la diferencia entre una investigación cuantitativa de una cualitativa a través de un ejemplo de cada una de ellas, elaboración de un mapa conceptual de la tesis y el cuadro de variables.

\section{Temas de reflexión}

Se incluye un tema de reflexión respecto de la ética y su relación con la investigación desarrollada.

\section{Tareas de avances en los capítulos}

Se elabora cada capítulo y se va realizando una revisión paulatina por parte de los tutores. Una vez revisada la tarea, el estudiante debe aplicar las sugerencias y correcciones solicitadas por el tutor.
Además, se realiza un borrador de los instrumentos de medición como parte de las tareas.

\section{Presentación de los \\ tres primeros capítulos}

Esta es una presentación oral ante el profesor tutor, con el fin de practicar tanto requisitos de forma como de fondo. Mediante esta tarea, el tutor verifica que el estudiante haya aplicado las correcciones solicitadas a lo largo de los capítulos y que pueda realizar su exposición con apoyo audiovisual, organizando las ideas, manejando adecuadamente el tiempo y con coherencia de análisis, entre otros aspectos.

\section{Informe final}

Se incorpora la realización de un informe final y una exposición del trabajo al concluir el tercer capítulo, es decir, el estudiante deberá exponer sus objetivos, así como un resumen de los marcos teórico y metodológico propuestos para su realización. Se agrega este elemento por la reiteración de comentarios de los alumnos en el sentido de que llegaban a presentar la defensa de la tesis sin haber tenido la posibilidad de realizar exposiciones orales durante toda su formación académica, precisamente por la modalidad a distancia. La exposición se 
realizará ante el tutor especialista y los demás educandos asignados al mismo tutor. En resumen, constituye una práctica para la defensa final de la tesis, con el fin de romper algunos de los paradigmas y limitantes de los alumnos a distancia, los cuales manifestaban temor por no contar con experiencias previas.
Análisis del impacto del nuevo diseño de conformidad con los estudiantes

De la opinión de los estudiantes consultados al finalizar el primer semestre del año 2011, se obtuvieron los siguientes resultados:

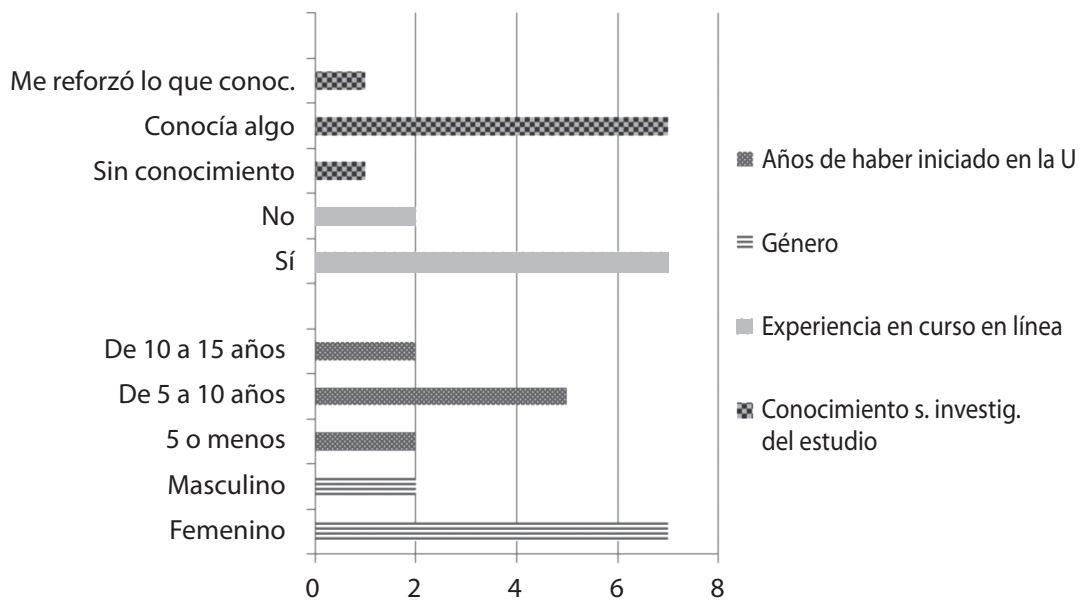

Figura 2. Perfil del estudiante. Fuente: Elaboración propia, con base en el cuestionario aplicado a los estudiantes del curso de Investigación Dirigida del primer semestre 2011.

Como se observa en el gráfico anterior, el mayor grupo de estudiantes en el nivel de licenciatura está representado por mujeres, y su permanencia en la Universidad oscila entre los cinco y diez años. Justamente por este motivo, la Escuela ha revisado los planes de estudio y -de manera colegiada- tomado las medidas necesarias para buscar el mejoramiento, la pertinencia, la calidad y, por último, la reducción de los tiempos de permanencia del estudiante; con ello, se busca aumentar, consecuentemente, el número de graduados en un promedio de tiempo menor, tal y como lo establecen los lineamientos de las instituciones responsables en el ramo (como SINAES Y CONARE). 
Por otro lado, es importante destacar que la iniciativa de la Escuela por incrementar los cursos en línea se ve reflejada en que la gran mayoría de estudiantes conoce el sistema y, por tanto, la plataforma les es familiar y accesible.

El desarrollo del curso 4010, tal cual está diseñado, no solo destaca por contribuir a la elaboración de los tres primeros capítulos de la tesis, sino también porque se refuerzan los conocimientos en metodología de investigación puesto que, de acuerdo con los datos presentados, en su mayoría nuestros educandos manifiestan conocer algo de investigación cuando concluyen sus programas de estudio.

La Cátedra de Investigación decidió migrar a la plataforma virtual con el fin de aprovechar la

\section{La plataforma virtual}

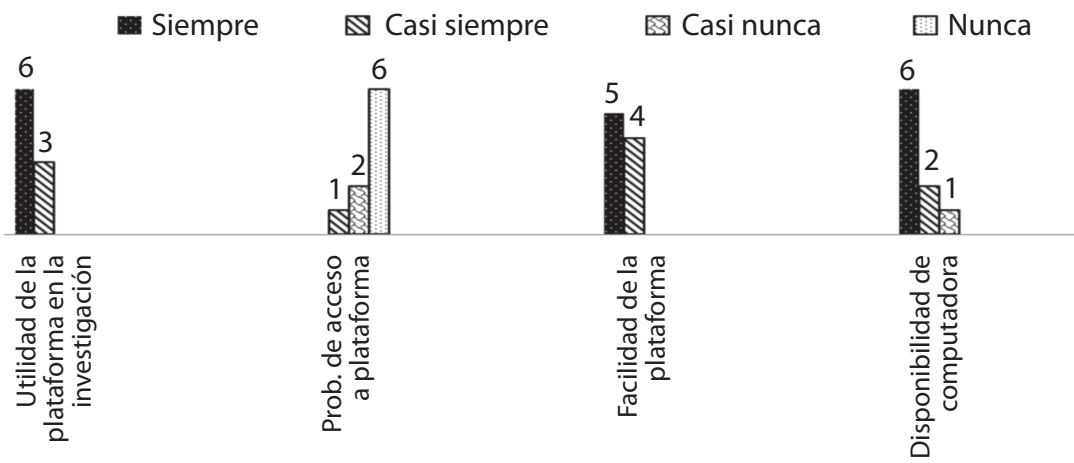

Figura 3. Uso de la plataforma virtual. Fuente: Elaboración propia, a partir del cuestionario.

tecnología y mejorar el acceso a la información. También se buscó mayor y mejor comunicación entre el coordinador, los tutores y estudiantes en el proceso de investigación, ofrecer más recursos de mediación, generar espacios de discusión e intercambio asincrónicos, entre otros motivos.

Según se observa en los resultados, la herramienta utilizada fue útil en el logro de los objetivos con alcances muy positivos.

El material de este curso estuvo representado por muchos años por el libro titulado Metodología de la investigación, de los autores Hernández, Fernández y Baptista (2010). Este texto es un excelente recurso, pues aporta conocimiento significativo en metodología; sin embargo, su aplicación práctica 
Impacto de los medios pedagógicos

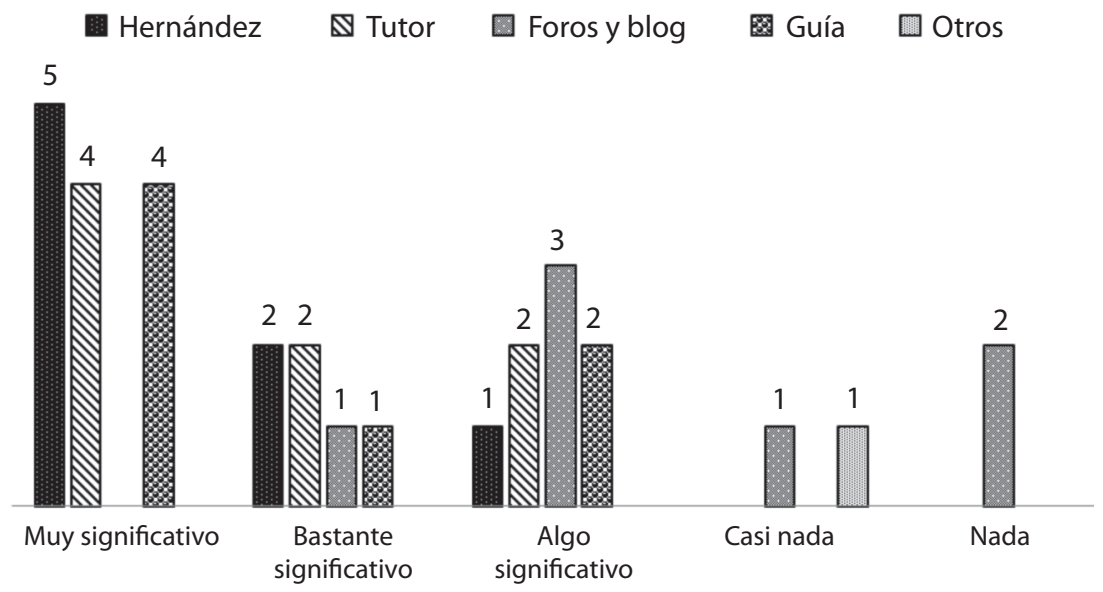

Figura 4. Mediación pedagógica. Fuente: Elaboración propia, a partir del cuestionario.

genera mucha dificultad o dudas por parte de los estudiantes. Por tal razón, se elaboró un material complementario, conocido como "Guía para la elaboración de la tesis", mediante el cual se orienta de principio a fin al educando en la elaboración de los apartados del informe final de graduación. Este manual ofrece orientación sobre aspectos administrativos que el estudiante debe acatar para presentar su trabajo de manera satisfactoria y cumplir con los requisitos de graduación que establece la Universidad; también, le brinda información sobre la estructura del trabajo, los requisitos formales y otros aspectos propios de la elaboración del proyecto.
Este material complementario fue utilizado, por primera vez, en el primer semestre del año 2011. En el mismo período de consulta, $y$ pese a que se encontraba en una versión preliminar, los estudiantes consideraron que este fue de gran utilidad y lo ubicaron en un segundo lugar en importancia, después del libro base.

El tutor especialista fue otro de los cambios realizados al curso -tal y como se indicó en un inicio$y$, de acuerdo con los datos, constituye un recurso de gran impacto, pues aporta conocimiento técnico, desde el inicio, que contribuye al estudiante a cumplir con la meta planteada y en un menor tiempo. 


\section{Intervención pedagógica}

288 Muy significativo

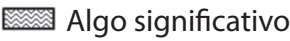

Wada significativo
Bastante significativo

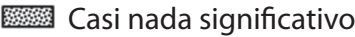

- Lineal (Muy significativo)

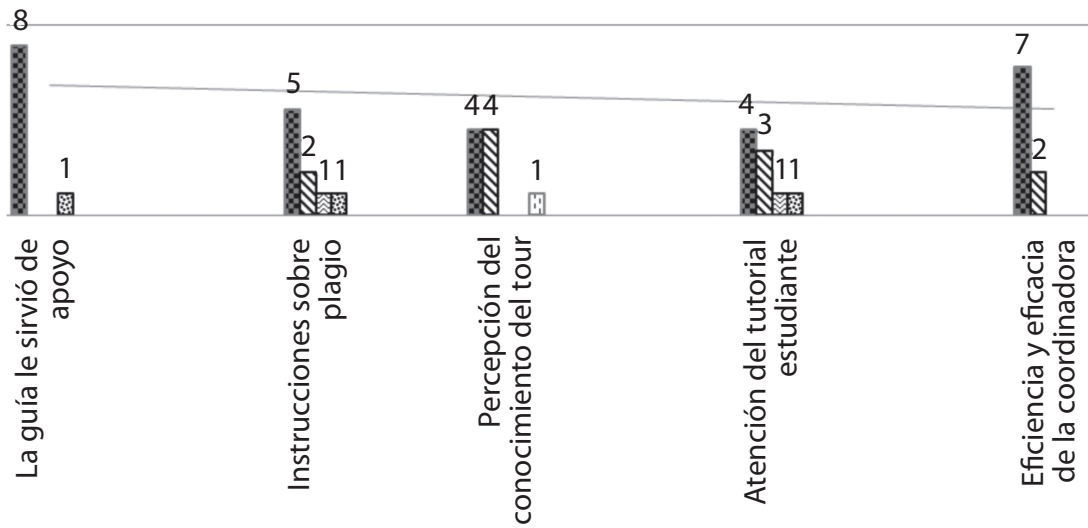

Figura 5. Mediación pedagógica. Fuente: Elaboración propia, a partir del cuestionario.

Dentro de los elementos innovadores en el diseño de este curso, se pueden mencionar: la creación de la figura del coordinador general y la asignación del especialista a cada estudiante, desde el inicio del proyecto. De acuerdo con los datos anteriores, ambos métodos de intervención pedagógica fueron significativos para los educandos. Es fundamental el aporte que se ofrece a través del material escrito, así como la información y coordinación del regulador del curso; al parecer así los perciben los encuestados quienes, en su mayoría, confirman que estos elementos junto con la orientación del tutor especialista fueron relevantes en el desarrollo de su trabajo y el cumplimiento de sus objetivos de estudio.

En resumen, se puede indicar que dos elementos para medir el cumplimiento de objetivos en el desarrollo de esta materia son: la realización de los tres primeros capítulos de la investigación y, por supuesto, el reforzamiento del conocimiento en esta materia. Al respecto, cabe señalar que un $70 \%$ de 


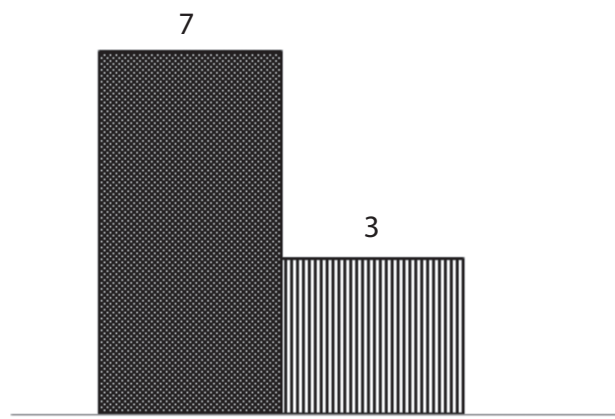

Totalmente satisfecho

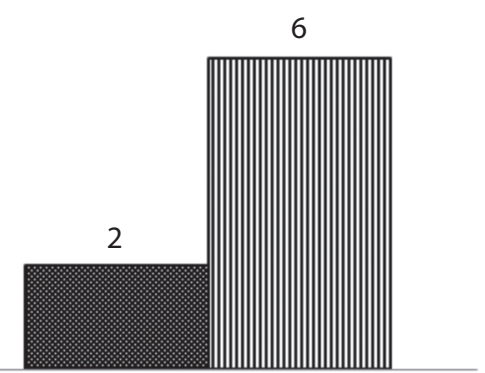

Bastante satisfecho

Figura 6. Expectativas. Fuente: Elaboración propia, a partir del cuestionario.

los alumnos que cursó la materia de Investigación Dirigida durante el periodo de estudio, alcanzó la meta propuesta de manera satisfactoria; lo cual es muy importante para el cumplimiento de objetivos de la Escuela. En general, los estudiantes perciben los cambios como positivos; no obstante, aún quedan retos por alcanzar y aspectos que mejorar.

\section{Conclusiones y recomendaciones}

En términos generales, luego de esta investigación, se puede concluir que los cambios efectuados por la Escuela de Ciencias de la Administración en el curso de
Investigación Dirigida, han tenido un impacto positivo; esto por cuanto se ha logrado fortalecer el área de investigación, la cual es prioritaria tanto como eje transversal de la Universidad, así como coyuntura histórica que se vive en el momento de acreditación de todos los énfasis que ofrece la carrera de Administración.

Uno de los objetivos alcanzados, a través de las modificaciones al diseño curricular de la materia 4010, es el favorecer la calidad de los trabajos finales de graduación y al mismo tiempo asegurarse de que el estudiante, en el plazo establecido, logre realizar su informe final de graduación con todos requisitos 
estipulados en el Reglamento de Trabajos Finales de Graduación. Con ello, contribuimos a reducir el plazo de permanencia de los estudiantes en la UNED, lo cual ha sido una preocupación institucional en los últimos años.

Cabe resaltar, también, que a través de la información ofrecida sobre el plagio y los mecanismos adecuados para evitarlo, se ha logrado disminuir la incidencia en los fraudes académicos o copia indiscriminada de documentos, que violentaban los derechos intelectuales de autor.

Un valor agregado de un diseño como este es el fortalecer el uso de todas las herramientas tecnológicas que ofrece la Universidad; asimismo, se busca la actualización de datos y contenido mediante la exploración de recursos como la biblioteca virtual, la base de datos internacionales, los préstamos interbibliotecarios; instrumentos que serán de utilidad para el desarrollo profesional de los egresados.

Una de las características de la educación a distancia es que limita la oportunidad de realizar trabajos de carácter presencial ante sus compañeros y profesores; por esta razón, en muchas oportunidades, el educando se enfrentaba a esta experiencia por primera vez en la defensa y presentación de su tesis. Con el fin de reducir este impacto, a través del curso se ofrece un espacio de práctica en la exposición del trabajo hecho hasta el momento, con lo cual se logra generar mayor confianza y mejor calidad en las presentaciones finales.

Gracias a los logros alcanzados, se enfrentan nuevos retos sobre todo cuando la Escuela de Ciencias de la Administración se sometió a un proceso de evaluación de la calidad de sus programas y recibió la visita de pares evaluadores, en setiembre del 2011. El resultado del informe de autoevaluación dado por los visitantes indica que una de las áreas que se debe fortalecer es la investigación en el nivel institucional, motivo por el cual se deben establecer coyunturas que permitan vincular la investigación de los egresados con las líneas de investigación previamente establecidas por la Universidad.

La Cátedra de Investigación es consciente de la responsabilidad que tiene en sus manos: la investigación es reflejo del desarrollo de un país y los profesionales, en los diversos campos, deben contar con las herramientas que les permitan desarrollarse en su campo profesional de una manera crítica.

\section{Referencias}

Bañuelos, A.M.; Barrón, H. (2005). Modelos de gestión del conocimiento para la educación en línea. Apertura. Año 5, núm. 1 (pp. 45-53) (nueva época). 
Cabero, J. (2006). Bases pedagógicas del elearning. Revista de Universidad y Sociedad del Conocimiento. Vol.3, No.1, pp. 1-10). Recuperado de: http://www. uoc.edu/rusc/3/1/dt/esp/cabero.pdf

Díaz, J. (2011). Modelos pedagógicos en la educación a distancia. Revista Electrónica de Humanidades, Educación y Comunicación Social, Vol. 12, pp. 86-113. Recuperado de: www.publicaciones.urbe.edu/index.php/REDHECS/.../300... - Venezuela

González-Videgaray, M.C. (2007). Evaluación de la redacción de alumnos y docentes en un modelo mixto de aprendizaje para educación superior. Revista Electrónica de Investigación y Evaluación Educativa. Vol. 13, núm.1, pp. 83-103. Recuperado de: http:// www.uv.es/RELIEVE/v13n1/RELIEVEv13n1_4.htm

Hernández, R.; Fernández, C. \& Baptista, P. (2010). Metodología de la investigación. México: Mc Graw Hill.

Llorente, M. C. (2006). El tutor de elearning: aspectos a tener en cuenta. Edutec. Revista Electrónica de Tecnología Educativa. No. 20, (p. 1-14). Recuperado de: http://edutec.rediris.es/ Revelec2/revelec20/1lorente.pdf 